\title{
Impacto do Trabalho em Profissionais de Serviços de Saúde Mental em um Município do Nordeste Brasileiro
}

\author{
Glaudênia Alves Moura Angelo Giuseppe Roncalli \\ Universidade Federal do Rio Grande do Norte, RN, Brasil. Universidade Federal do Rio Grande do Norte, RN, Brasil. \\ Luiz Roberto Augusto Noro \\ Universidade Federal do Rio Grande do Norte, RN, Brasil.
}

\begin{abstract}
Resumo: O objetivo deste estudo foi avaliar o impacto laboral sentido pelos profissionais de saúde, em virtude do trabalho diário com pessoas que apresentam distúrbios psiquiátricos. Trata-se de uma pesquisa de campo, numa abordagem quantitativa. A coleta de dados ocorreu por meio da Escala de Avaliação do Impacto do Trabalho em Serviços de Saúde Mental aplicada em 87 profissionais de Saúde Mental da cidade de Mossoró, Brasil. Os resultados apontam baixo efeito de sobrecarga nos profissionais pesquisados. Das subescalas estudadas, a maior pontuação foi observada na que mede o impacto do trabalho no funcionamento da equipe. Pôde-se concluir que as relações interativas entre os profissionais e os usuários não são as causas de maior impacto no trabalho.
\end{abstract}

Palavras-chave: Serviços de saúde mental, Saúde do trabalhador, Recursos humanos, Psicologia.

\section{Work impact on professionals from mental health services in a Brazilian northeast city}

\begin{abstract}
The aim of this study was to evaluate the work impact felt by health professionals dealing with people who have psychiatric disorders. This is a field research on a quantitative approach. Data collection occurred through the Impact Assessment for Mental Health Services tool applied to 87 mental health professionals in the city of Natal, Brazil. The results show a low overhead effect on the professionals surveyed. In the subscales studied, the highest score was observed in measuring the impact of work on the functioning of the team. It was concluded that the interactive relationships between professionals and users are not the cause of greater impact on the work.
\end{abstract}

KeYwords: Mental health services, Occupational health, Human resources, Psychology. 


\title{
Impacto laboral sobre profesionales de servicios de salud mental en una ciudad del nordeste brasileño
}

\begin{abstract}
Resumen: El objetivo de este estudio fue evaluar el impacto laboral sentido por profesionales de la salud, asociado a su trabajo diario con personas que tienen trastornos psiquiátricos. Se trata de una investigación de campo con enfoque cuantitativo. Los datos fueron recolectados a través de la Evaluación de Impacto de los Servicios de Salud Mental, aplicada a 87 profesionales de salud mental de la ciudad de Natal, Brasil. Los resultados muestran un efecto bajo de sobrecarga en los profesionales encuestados. En las subescalas estudiadas, la mayor puntuación observada correspondió a la medición del impacto laboral sobre el funcionamiento del equipo. Se concluyó que las relaciones interactivas entre profesionales y usuarios no son las causas de mayor impacto en el trabajo.
\end{abstract}

Palabras clave: Servicios de salud mental, Salud laboral, Recursos humanos, Psicología.

\section{Introdução}

O Sistema Único de Saúde brasileiro configura-se como um campo em permanente construção, em especial por seu desafio de proporcionar saúde para toda a população brasileira de forma integral e gratuita, a partir de política econômica compatível, constituindo um fator de democratização e distribuição equitativa dos recursos aplicados (Amoretti, 2005).

No campo da Saúde Mental, esta reorganização da atenção apontou a inserção de outros saberes, além da presença de outros profissionais (Nascimento, \& Galvanese, 2009), caracterizando-se por profundas modificações na assistência às pessoas com transtornos mentais e de comportamento, caracterizada pela Reforma Psiquiátrica (Ballarin, Carvalho, Ferigato, Miranda, \& Magaldi, 2011). Segundo a Organização Mundial da Saúde, é crescente o número de pessoas acometidas por transtornos mentais e de comportamento, o que exige investimento financeiro na transformação dos espaços assistenciais assim como na formação de recursos humanos (Onocko-Campos, \& Furtado, 2006).

Como fruto de um processo de desinstitucionalização e de uma nova prática de cuidados em saúde mental, surgiu no Brasil o primeiro Centro de Atenção Psicossocial (CAPS), na cidade de São Paulo, em 1987 (Brasil, 2005; Tenório, 2002), o qual se constitui como importante estratégia do processo de Reforma Psiquiátrica. Os CAPS são instituições destinadas a acolher pessoas com transtornos mentais, buscando integrá-las a um ambiente social, cultural e familiar, bem como as apoiando nas suas iniciativas e autonomias, oferecendo-lhes cuidados clínicos e de reabilitação psicossocial, evitando internações e favorecendo o exercício da cidadania dos usuários e seus familiares (Brasil, 2004).

Essa forma de cuidar exige dos profissionais um maior envolvimento com a clientela assistida, contribuindo para um processo terapêutico mais próximo e mais coerente com princípios do cuidado em saúde. Entretanto, segundo alguns autores (Bandeira, Ishara, \& Zuardi, 2007; Rebouças, Abelha, Legay, \& Lovisi, 2008), para que este vínculo se viabilize, é fundamental a adoção de políticas públicas direcionadas ao bem-estar e à qualidade de saúde dos profissionais, caso contrário a baixa satisfação com o trabalho e a sobrecarga sentida podem afetar a qualidade dos atendimentos prestados.

Como princípios e diretrizes norteadores dessa politica, há a valorização do trabalhador, garantindo a sua qualificação continuada, a remuneração justa e condições de trabalho adequadas, a implantação de planos de cargos, carreiras e salários, a democratização das relações e discussões em todos os níveis de gestão, a incorporação da segurança, saúde e saúde mental do trabalhador, a supervisão clínica e institucional, assim como a garantia da jornada de trabalho adequada destes profissionais (Brasil, 2001a).

Considerando a situação atual, o presente estudo teve como objetivo avaliar a sobrecarga sentida pelos profissionais de saúde, em virtude do trabalho diário 
com pessoas que apresentam distúrbios mentais, uma vez que essa sobrecarga pode vir a afetar a sua saúde física e emocional, interferindo na qualidade dos serviços oferecidos.

\section{Método}

Trata-se de uma pesquisa de campo, com abordagem quantitativa. A população do estudo foi constituída por trabalhadores de todas as instituições da Rede de Saúde Mental do município de Mossoró-RN, composta por quatro CAPS e uma Unidade Integrada de Saúde Mental, todas inscritas no Cadastro Nacional dos Estabelecimentos de Saúde (Brasil, 2012). Como critério de inclusão, foi considerada a necessidade de que esses trabalhadores possuíssem vínculo empregatício com a instituição analisada e que tivessem exercido atividades diretamente relacionadas à assistência às pessoas com transtornos emocionais, sendo excluídos os trabalhadores voluntários e estagiários.

\section{Instrumento de medida}

Foi utilizada a Escala de Avaliação do Impacto do Trabalho em Serviços de Saúde Mental (Impacto-BR), a qual avalia a sobrecarga sentida pelos profissionais de saúde mental em consequência do trabalho diário com pessoas portadoras de distúrbios psiquiátricos (Bandeira, Ishara, \& Zuardi, 2007). A tradução e adaptação da Escala Impacto para o Brasil foram desenvolvidas pelo Laboratório de Investigações em Saúde Mental da Universidade de São Paulo, enquanto a análise e a validação foram obtidas no Laboratório de Pesquisa em Saúde Mental da Universidade Federal de São João Del Rei, compondo parte de um projeto multicêntrico da OMS (Pitta, 1996; Bandeira, Pitta, \& Mercier, 2000).

A Escala Impacto-BR é constituída por 34 itens: seis relativos às variáveis sociodemográficas (idade, sexo, estado civil, nível de escolaridade, profissão e tempo de trabalho em saúde mental); 11 itens que visam obter informações complementares (local, horário, tempo de trabalho em saúde mental, jornada semanal, tipo de atividade desenvolvida com o usuário do serviço e repercussões do trabalho em saúde mental nos sentimentos de culpabilidade, no nível de estresse ressentido, na necessidade de apoio profissional, na noção de satisfação profissional e na atitude da família do profissional em relação ao seu trabalho); 18 itens que compõem a Escala Global a qual avalia o grau de sobrecarga sentida pela equipe, a partir de uma escala ordinal do tipo Likert de cinco pontos (quanto menor o valor, menor o impacto do trabalho).

Ositens que compõem a Escala Global constam de duas questões que se referem ao impacto do trabalho na vida social e no relacionamento familiar do profissional (Impacto Geral) e três fatores: o Fator 1 contém cinco itens que avaliam o impacto do trabalho sobre a saúde física e mental do próprio profissional de saúde; o Fator 2 contém seis itens que avaliam o impacto do trabalho sobre o funcionamento da equipe e o Fator 3 contém cinco itens que avaliam os efeitos do trabalho no estado emocional do profissional.

\section{Procedimentos de coleta de dados}

Por meio de uma reunião com o coordenador de cada uma das instituições participantes do estudo, os objetivos da pesquisa foram apresentados pelo pesquisador e discutidos com todos os profissionais da Unidade, tanto os de nível superior (enfermeiros, médicos, educadores físicos, assistentes sociais, terapeutas ocupacionais, psicólogos, pedagogos e fonoaudiólogos) quanto os de nível médio (técnicos de enfermagem) e fundamental (agentes administrativos, recepcionistas, auxiliares de serviços gerais e cozinheiras).

Em seguida, os profissionais que se propuseram a participar do estudo assinaram o Termo de Consentimento Livre e Esclarecido e, a partir daí, receberam instruções relativas ao preenchimento da Escala Impacto-BR. A escala é um instrumento autoadministrado e foi respondida individualmente e entregue ao pesquisador responsável pelo sigilo das informações prestadas.

\section{Consolidação e análise dos dados}

A construção do banco de dados e sua posterior análise ocorreram no programa SPSS 17.1. Foi feita uma análise descritiva dos dados, apresentando suas frequências e medidas de dispersão. $\mathrm{O}$ cálculo do grau de sobrecarga geral da equipe no serviço de saúde mental foi aferido por meio da média das respostas obtidas nas questões que compõem a escala, assim como cada um dos três fatores. Foi realizada análise descritiva dos dados com suas frequências e medidas de dispersão, assim como foi utilizado o teste do Qui-quadrado para verificar possíveis associações. 


\section{Aspectos éticos}

O Projeto de Pesquisa foi submetido à apreciação do Comitê de Ética em Pesquisa do Hospital Universitário Onofre Lopes da Universidade Federal do Rio Grande do Norte e aprovado pelo Parecer $\mathrm{n}^{\circ} 16.563$, em 29 de abril de 2012. A pesquisa não apresentou riscos físicos aos participantes.

\section{Resultados}

Participaram do estudo 87 profissionais, com idade média de 43,2 anos ( $D P=9,6$ ), sendo que a idade mínima foi de 25 anos e a máxima de 64 anos. A maioria é composta por pessoas do sexo feminino (75,9\%), casadas ou vivendo com companheiros $(66,7 \%)$. Em relação ao perfil do cargo, 56,3\% trabalhavam em cargos de nível superior, $48,3 \%$ são pós-graduados, $87,4 \%$ trabalhavam em um único serviço de saúde mental, 59,8\% trabalhavam em dois turnos ou mais. Quanto à experiência em saúde mental, 56,3\% atuam nessa área há seis anos ou mais. Em relação à sobrecarga de trabalho dos profissionais, são apresentados na Tabela 1 os valores dos escores globais dos fatores e das subescalas da Impacto-BR.

$\mathrm{Na}$ análise descritiva da escala Impacto-BR, observa-se um baixo efeito de sobrecarga entre os profissionais pesquisados, uma vez que o impacto geral observado foi de 1,48 (DP = 0,716), em escala variando de 1 a 5 .

A Tabela 2 refere-se à média e mediana dos escores da subescala Fator 1 (impacto sobre a saúde física e mental), relacionando-se às características funcionais e sociais dos participantes.

No tocante ao impacto sobre a saúde física e emocional dos profissionais, constata-se maior pontuação entre profissionais de nível superior, que trabalham em um único serviço, do sexo feminino e com menor faixa etária. Esses valores, entretanto, não apresentaram diferença estatisticamente significativa.

A Tabela 3 demonstra a relação entre o Fator 2 e as características sociais e funcionais dos participantes, a partir das médias e medianas dos escores.

Tabela 1

Análise descritiva da escala Impacto-BR/subescalas em termos de médias, desvio-padrão, valor mínimo e valor máximo, assim como o impacto geral.

\begin{tabular}{lcccc}
\hline Escala Impacto-BR & Média & Desvio-padrão & Valor mínimo & Valor máximo \\
\hline Fator 1 (Saúde fisica e mental) & 1,35 & 0,660 & - & - \\
Aumento de queixas físicas & 1,61 & 0,753 & 1 & 4 \\
Aumento necessidade de procurar médico & 1,29 & 0,608 & 1 & 4 \\
Uso de mais medicação & 1,25 & 0,686 & 1 & 5 \\
Trabalho afetando a estabilidade emocional & 1,40 & 0,739 & 1 & 4 \\
Precisou ajuda profissional saúde mental & 1,22 & 0,515 & 1 & 3 \\
Fator 2 (Funcionamento da equipe) & 1,62 & 0,847 & - & - \\
Receio de ser agredido & 2,17 & 0,892 & 1 & 4 \\
Trabalho afetando o estado de saúde física & 1,44 & 0,694 & 1 & 3 \\
Afastamento por estresse & 1,40 & 0,739 & 1 & 4 \\
Presença de distúrbio do sono & 1,41 & 0,883 & 1 & 5 \\
Sentimento de ter outro tipo de emprego & 1,62 & 1,040 & 1 & 5 \\
Pensa em mudar de campo de trabalho & 1,75 & 1,940 & 1 & 5 \\
Fator 3 (Repercussões emocionais) & 1,60 & 0,775 & - & - \\
Sentimento de frustração & 1,64 & 0,902 & 1 & 5 \\
Sentimento de sobrecarga & 1,62 & 0,796 & 1 & 4 \\
Sentimento de cansaço físico & 2,17 & 0,943 & 1 & 5 \\
Sentimento de depressão & 1,13 & 0,426 & 1 & 3 \\
Sentimento de estresse & 1,41 & 0,708 & 1 & 4 \\
Impacto geral & 1,48 & 0,716 & - & - \\
Trabalho interfere relacionamento familiar & 1,06 & 0,234 & 1 & 2 \\
Trabalho afetando a vida pessoal & 1,13 & 0,501 & 1 & 4 \\
\hline
\end{tabular}


Tabela 2

Escores do Fator 1 em relação às características funcionais e sociais em profissionais de saúde mental $(n=87)$.

\begin{tabular}{|c|c|c|c|c|c|c|}
\hline Características & $\mathrm{n}$ & Média & DP & Mediana & L.I.* & L.S. ${ }^{* *}$ \\
\hline \multicolumn{7}{|l|}{ Cargo } \\
\hline Superior & 50 & 1,343 & 0,372 & 1,200 & 1,239 & 1,447 \\
\hline Médio/Fundamental & 37 & 1,389 & 0,553 & 1,000 & 1,207 & 1,571 \\
\hline \multicolumn{7}{|l|}{ Tipo de serviço } \\
\hline CAPS $* * *$ & 65 & 1,342 & 0,450 & 1,200 & 1,232 & 1,451 \\
\hline Ambulatório & 22 & 1,391 & 0,480 & 1,200 & 1,190 & 1,591 \\
\hline \multicolumn{7}{|l|}{ Quantidade de serviços } \\
\hline Trabalho único & 76 & 1,387 & 0,474 & 1,200 & 1,280 & 1,493 \\
\hline Trabalho em dois ou mais & 11 & 1,127 & 0,185 & 1,000 & 1,018 & 1,237 \\
\hline \multicolumn{7}{|l|}{ Carga horária total } \\
\hline Mais de 30h/semanal & 40 & 1,335 & 0,484 & 1,200 & 1,185 & 1,485 \\
\hline Menos de 30h/semanal & 47 & 1,370 & 0,433 & 1,200 & 1,246 & 1,494 \\
\hline \multicolumn{7}{|l|}{ Tipo de atividade } \\
\hline Grupal & 33 & 1,358 & 0,458 & 1,200 & 1,201 & 1,514 \\
\hline Individual & 54 & 1,352 & 0,458 & 1,200 & 1,230 & 1,474 \\
\hline \multicolumn{7}{|l|}{ Quantidade de turnos } \\
\hline Único turno & 35 & 1,354 & 0,431 & 1,200 & 1,211 & 1,497 \\
\hline Dois ou mais turnos & 52 & 1,354 & 0,475 & 1,200 & 1,225 & 1,483 \\
\hline \multicolumn{7}{|l|}{ Faixa etária } \\
\hline 25 a 39 anos & 38 & 1,432 & 0,484 & 1,400 & 1,278 & 1,586 \\
\hline 40 anos ou mais & 49 & 1,294 & 0,426 & 1,000 & 1,775 & 1,413 \\
\hline \multicolumn{7}{|l|}{ Sexo } \\
\hline Masculino & 21 & 1,219 & 0,340 & 1,000 & 1,074 & 1,364 \\
\hline Feminino & 66 & 1,397 & 0,480 & 1,200 & 1,281 & 1,513 \\
\hline \multicolumn{7}{|l|}{ Tempo de atuação } \\
\hline Menos de seis anos & 38 & 1,390 & 0,530 & 1,200 & 1,221 & 1,558 \\
\hline Seis anos ou mais & 49 & 1,327 & 0,391 & 1,200 & 1,217 & 1,436 \\
\hline
\end{tabular}

* L.I.: Limite inferior.

** L.S.: Limite superior.

*** CAPS: Centro de Atenção Psicossocial.

Em relação ao impacto sobre o funcionamento da equipe, os dados apontam que os profissionais com cargos de nível médio, trabalhadores de CAPS, profissionais que atuam em um único serviço e que desenvolvem atividades em grupo e em um único turno sentem mais esse impacto, assim como os profissionais do sexo feminino e com menor tempo de atuação.

A Tabela 4 refere-se à média e mediana dos escores da subescala Fator 1 (impacto sobre o estado emocional dos profissionais), relacionando-se às características funcionais e sociais dos participantes.

O fator 3 aborda o sentimento de sentir-se frustrado, sobrecarregado, estressado, depressivo com os resultados do seu trabalho, além da sensação de cansaço após o trabalho. Quanto à subescala que mede o impacto sobre o estado emocional dos profissionais, constatam-se maiores pontuações em trabalhadores com cargos de nível médio, atuantes em ambulatórios de saúde mental com mais de 30 horas semanais, com menor faixa etária e menos tempo de atuação. 
Tabela 3

Escores do Fator 2 em relação às características funcionais e sociais em profissionais de saúde mental (n=87).

\begin{tabular}{|c|c|c|c|c|c|c|}
\hline Características & $\mathrm{n}$ & Média & DP & Mediana & L.I. ${ }^{*}$ & L.S. ${ }^{* *}$ \\
\hline \multicolumn{7}{|l|}{ Cargo } \\
\hline Superior & 50 & 1,554 & 0,431 & 1,500 & 1,434 & 1,675 \\
\hline Médio/Fundamental & 37 & 1,722 & 0,420 & 1,750 & 1,585 & 1,859 \\
\hline \multicolumn{7}{|l|}{ Tipo de serviço } \\
\hline CAPS ${ }^{* * *}$ & 65 & 1,633 & 0,442 & 1,667 & 1,526 & 1,741 \\
\hline Ambulatório & 22 & 1,576 & 0,407 & 1,417 & 1,406 & 1,746 \\
\hline \multicolumn{7}{|l|}{ Quantidade de serviços } \\
\hline Trabalho único & 76 & 1,654 & 0,441 & 1,667 & 1,554 & 1,753 \\
\hline Trabalho em dois ou mais & 11 & 1,379 & 0,270 & 1,333 & 1,219 & 1,538 \\
\hline \multicolumn{7}{|l|}{ Carga horária total } \\
\hline Mais de 30h/semanal & 40 & 1,638 & 0,464 & 1,667 & 1,494 & 1,781 \\
\hline Menos de 30h/semanal & 47 & 1,603 & 0,408 & 1,500 & 1,486 & 1,719 \\
\hline \multicolumn{7}{|l|}{ Tipo de atividade } \\
\hline Grupal & 33 & 1,682 & 0,455 & 1,667 & 1,526 & 1,837 \\
\hline Individual & 54 & 1,508 & 0,417 & 1,500 & 1,469 & 1,691 \\
\hline \multicolumn{7}{|l|}{ Quantidade de turnos } \\
\hline Único turno & 35 & 1,614 & 0,406 & 1,667 & 1,480 & 1,749 \\
\hline Dois ou mais turnos & 52 & 1,622 & 0,453 & 1,583 & 1,499 & 1,745 \\
\hline \multicolumn{7}{|l|}{ Faixa etária } \\
\hline 25 a 39 anos & 38 & 1,772 & 0,437 & 1,667 & 1,633 & 1,911 \\
\hline 40 anos ou mais & 49 & 1,500 & 0,392 & 1,333 & 1,390 & 1,610 \\
\hline \multicolumn{7}{|l|}{ Sexo } \\
\hline Masculino & 21 & 1,579 & 0,521 & 1,500 & 1,357 & 1,802 \\
\hline Feminino & 66 & 1,631 & 0,404 & 1,667 & 1,534 & 1,729 \\
\hline \multicolumn{7}{|l|}{ Tempo de atuação } \\
\hline Menos de seis anos & 38 & 1,706 & 0,437 & 1,667 & 1,567 & 1,845 \\
\hline Seis anos ou mais & 49 & 1,551 & 0,420 & 1,500 & 1,433 & 1,669 \\
\hline
\end{tabular}

* L.I.: Limite inferior.

** L.S.: Limite superior.

*** CAPS: Centro de Atenção Psicossocial.

De acordo com os resultados apresentados, observa-se que os profissionais que trabalham em um único serviço, em relação aos que trabalham em dois ou mais serviços, apresentam maior impacto no fator geral, fator 1 e fator 2 .

Destaca-se também que os profissionais com idade entre 25 e 39 anos apresentam maior impacto em todos os fatores da escala que o verificado naqueles profissionais maiores de 40 anos. E ainda que os profissionais com menor tempo de atuação nos serviços apresentam pontuações maiores no fator geral, fator 2 e fator 3, quando comparados aos que possuem maior experiência.

Quando analisado isoladamente, o Fator 2, que corresponde aos efeitos no funcionamento da equipe foi o que obteve maior índice $(1,62)$. Entretanto, esse resultado também indica baixo efeito de sobrecarga entre os profissionais pesquisados. No Fator 2, o item que demonstrou maior índice $(2,17)$ foi o receio de ser agredido por um paciente. A Tabela 5 apresenta os resultados deste item em relação ao nível de cargo exercido e o tempo de atuação nas instituições pesquisadas. 
Tabela 4

Escores do Fator 3 em relação às características funcionais e sociais em profissionais de saúde mental ( $n=87)$.

\begin{tabular}{|c|c|c|c|c|c|c|}
\hline Características & $\mathrm{n}$ & Média & $\mathrm{DP}$ & Mediana & L.I. ${ }^{*}$ & L.S.** \\
\hline \multicolumn{7}{|l|}{ Cargo } \\
\hline Superior & 50 & 1,563 & 0,448 & 1,400 & 1,438 & 1,689 \\
\hline Médio/Fundamental & 37 & 1,656 & 0,491 & 1,500 & 1,495 & 1,816 \\
\hline \multicolumn{7}{|l|}{ Tipo de serviço } \\
\hline CAPS $* * *$ & 65 & 1,560 & 0,430 & 1,400 & 1,455 & 1,665 \\
\hline Ambulatório & 22 & 1,700 & 0,555 & 1,500 & 1,468 & 1,932 \\
\hline \multicolumn{7}{|l|}{ Quantidade de serviços } \\
\hline Trabalho único & 76 & 1,618 & 0,468 & 1,400 & 1,513 & 1,724 \\
\hline Trabalho em dois ou mais & 11 & 1,436 & 0,437 & 1,400 & 1,178 & 1,694 \\
\hline \multicolumn{7}{|l|}{ Carga horária total } \\
\hline Mais de 30h/semanal & 40 & 1,595 & 0,438 & 1,600 & 1,459 & 1,731 \\
\hline Menos de 30h/semanal & 47 & 1,596 & 0,493 & 1,400 & 1,455 & 1,737 \\
\hline \multicolumn{7}{|l|}{ Tipo de atividade } \\
\hline Grupal & 54 & 1,619 & 0,523 & 1,400 & 1,479 & 1,758 \\
\hline Individual & 33 & 1,558 & 0,356 & 1,400 & 1,436 & 1,679 \\
\hline \multicolumn{7}{|l|}{ Quant. Turnos } \\
\hline Único turno & 35 & 1,611 & 0,502 & 1,400 & 1,445 & 1,778 \\
\hline Dois ou mais turnos & 52 & 1,585 & 0,444 & 1,400 & 1,464 & 1,705 \\
\hline \multicolumn{7}{|l|}{ Faixa etária } \\
\hline 25 a 39 anos & 38 & 1,663 & 0,449 & 1,600 & 1,521 & 1,806 \\
\hline 40 anos ou mais & 49 & 1,543 & 0,476 & 1,400 & 1,410 & 1,676 \\
\hline \multicolumn{7}{|l|}{ Sexo } \\
\hline Masculino & 21 & 1,505 & 0,403 & 1,600 & 1,332 & 1,677 \\
\hline Feminino & 66 & 1,624 & 0,483 & 1,400 & 1,508 & 1,741 \\
\hline \multicolumn{7}{|l|}{ Tempo de atuação } \\
\hline Menos de seis anos & 38 & 1,621 & 0,485 & 1,600 & 1,467 & 1,775 \\
\hline Seis anos ou mais & 49 & 1,576 & 0,454 & 1,400 & 1,448 & 1,703 \\
\hline $\begin{array}{l}{ }^{*} \text { L.I.: Limite inferior. } \\
\text { ** L.S.: Limite superior. } \\
{ }^{* * *} \text { CAPS: Centro de Atenção Psice }\end{array}$ & & & & & & \\
\hline \multicolumn{7}{|c|}{ Tabela 5} \\
\hline Receio ser agredido & Nenhum & forma & Não muito & Mais ou menos & Muito & Total \\
\hline \multicolumn{7}{|l|}{ Nível } \\
\hline Superior & 18 & & 18 & 14 & 0 & 50 \\
\hline Médio/Fundamental & 6 & & 10 & 17 & 4 & 37 \\
\hline \multicolumn{7}{|l|}{ Tempo de atuação } \\
\hline Menos de seis anos & 8 & & 10 & 18 & 2 & 38 \\
\hline Seis anos ou mais & 16 & & 18 & 13 & 2 & 49 \\
\hline
\end{tabular}


Observa-se que esse sentimento aparece em todos os níveis da escala, nos cargos de nível médio/técnico/fundamental, ocupados pelos agentes administrativos, recepcionistas e técnicos de enfermagem. O receio de ser agredido surge com mais frequência nos profissionais com menor tempo de atuação, todavia, não desaparece ao longo da experiência na área de saúde mental.

\section{Discussão}

A presente pesquisa buscou avaliar a sobrecarga sentida pelos profissionais de saúde mental em função do seu trabalho diário com pessoas acometidas por transtornos psiquiátricos, uma vez que o trabalho em saúde mental é considerado por alguns autores (Santos, \& Cardoso, 2010) de acentuada vulnerabilidade a sobrecarga emocional.

Os resultados encontrados no presente estudo, entretanto, corroboram com outras pesquisas realizadas anteriormente (Camilo, Bandeira, Leal, \& Scalon, 2012; Santos, Cardoso, \& Vieira, 2011; Pelisoli, Moreira, \& Kristensen, 2007; Leal, Bandeira, \& Azevedo, 2012), uma vez que foi observado baixo impacto de sobrecarga sentida pelos profissionais de saúde mental em consequência do trabalho diário com pessoas portadoras de distúrbios psiquiátricos, com um escore médio de 1,48 (escala de 1 a 5). Não foi observada relação entre impacto do trabalho e as variáveis sexo, faixa etária, grau de instrução relacionado ao cargo, tipo de serviço e de atividade, carga horária, número de vínculos empregatícios, tempo de atuação no serviço público e quantidade de turnos.

Apesar dos escores considerados baixos, o que apresentou maior impacto $(1,62)$ foi o relativo ao impacto do trabalho no funcionamento da equipe. De acordo com Sampaio, Guimarães, Carneiro, \& Garcia Filho (2011), o trabalho interdisciplinar surge como consequência da complexidade do conhecimento, sinalizando a necessidade dos trabalhadores se relacionarem entre si, com os gestores, com os usuários e seus familiares, na perspectiva da construção coletiva de um projeto terapêutico. Contrapondo-se a essa visão, Vasconcellos (2010) ressalta que a tendência ao individualismo, a dificuldade em transferir conhecimentos teóricos de cada saber para um trabalho coletivo, as representações dos outros profissionais/saberes na forma de ameaça favorecem a não comunicação, o que pode vir a alimentar conflitos profissionais.
Observa-se que $56,3 \%$ dos profissionais participantes deste estudo estão há seis anos ou mais trabalhando nessa área e que 57,5\% nunca pensaram em mudar de campo de trabalho; $66,7 \%$ acreditam que ter outro tipo de emprego não os faria sentir-se mais saudáveis emocionalmente e $82,8 \%$ afirmaram que nunca precisaram de ajuda de outro profissional de saúde mental. Porém, isso não significa que não haja nenhum sofrimento ou desgaste emocional, uma vez que, para os profissionais de saúde, o contato direto com outros indivíduos doentes impõe necessariamente o contato com sua própria vida, saúde e doença, com seus próprios conflitos. O manejo dessas situações dependerá de recursos subjetivos, de mecanismos de defesa que permitam ter uma adequada adaptação profissional. Todavia, esses mecanismos de defesa não podem ser tão rígidos para não levar a uma inadequada adaptação profissional, acarretando padrões fixos de atitudes e práticas diante dos usuários dos serviços, prejudicando assim a qualidade da assistência (Brasil, 2001b).

Em todas as instituições pesquisadas, há algum profissional que apresenta receio de ser agredido por algum paciente. Essa informação surge também no trabalho realizado por Camilo et al. (2012), no qual o receio de ser agredido por um paciente e a sensação de cansaço físico obteve maior percentual dentre os itens da escala.

Nesse caso, nota-se que esse sentimento é mais frequente nos cargos de nível médio/técnico/fundamental. Fazem parte desses cargos os agentes administrativos, recepcionistas e técnicos de enfermagem. Os agentes administrativos e recepcionistas são geralmente aqueles profissionais que estão na chamada linha de frente do serviço, ou ainda na porta de entrada, recebendo os usuários e seus familiares. São os responsáveis em passar as primeiras informações, organizar a agenda e a marcação de consultas dos profissionais, dentre outras atividades, lidando tanto com o público externo quanto com os outros profissionais do serviço. Os técnicos de enfermagem, por sua vez, dentre outras funções, acabam sendo os profissionais responsáveis por conter os usuários mais agitados, por vezes ministrando alguma medicação. Tais circunstâncias podem levar a uma maior sobrecarga entre esses profissionais e a uma maior sensação de cansaço físico nessas categorias.

De acordo com os dados coletados, o receio de ser agredido surge com mais frequência nos profissionais com menor tempo de atuação. Todavia, não desaparece ao longo da experiência na área de saúde 
mental, indicando que possivelmente os profissionais trabalhem num estado de alerta defensivo quanto a uma provável agressão por parte dos usuários dos serviços. Apesar de não haver diferenças estatisticamente significativas entre as análises realizadas, observa-se que, semelhantemente a estudos anteriores, quanto maior a idade e o tempo de atuação, menor o impacto emocional no trabalho, o que sugere que a experiência aumenta a segurança nas decisões tomadas e a possibilidade de um maior controle sobre as demandas de trabalho, diminuindo o estresse e a exaustão emocional (De Marco, Citero, Moraes, \& Nogueira-Martins, 2008).

\section{Referências}

Amoretti, R. (2005). A educação médica diante das necessidades sociais em saúde. Revista Brasileira de Educação Médica, 29(2), 136-146. Recuperado de http:// www2.ghc.com.br/ghc/Noticias/Not071105_01.pdf

Ballarin, M. L. G. S., Carvalho, F. B., Ferigato, S. H., Miranda I. M. S., \& Magaldi C. C. (2011). Centro de Atenção Psicossocial: convergência entre a saúde mental e a saúde coletiva. Psicologia em Estudo, 16(4), 603-611.

Bandeira, M., Ishara, S., \& Zuardi, A. (2007). Satisfação e sobrecarga de profissionais de saúde mental: validade de construto das escalas SATIS-BR e IMPACTO-BR. Jornal Brasileiro de Psiquiatria, 56(4), 280-286. doi:10.1590/S0047-20852007000400007

Bandeira, M., Pitta, A. M. F., \& Mercier, C. (2000). Escalas brasileiras de avaliação da satisfação (SATIS-BR) e da sobrecarga (IMPACTO-BR) da equipe técnica em serviços de saúde mental. Jornal Brasileiro de Psiquiatria, 49(4), 105-115.

Brasil. (2012). Cadastro Nacional de Estabelecimentos de Saúde. Recuperado de http://cnes.datasus.gov.br

Brasil. (2001b). Lei no 10.216, de 06 de abril de 2001. Dispõe sobre a proteção e os direitos das pessoas portadoras de transtornos mentais e redireciona o modelo assistencial em saúde mental. Diário Oficial da União. 9 abril.

Brasil. (2001a). Ministério da Saúde. Secretaria de Assistência à Saúde. Programa Nacional de Humanização da Assistência Hospitalar. Brasília, DF: Ministério da Saúde.

Brasil. (2004). Ministério da Saúde. Secretaria de Atenção a Saúde Departamento de Ações Programáticas Estratégicas. Saúde mental no SUS: os centros de atenção psicossocial (Série F: Comunicação e Educação em Saúde). Brasília, DF: o autor.

\section{Conclusões}

O trabalho apresentado demonstrou que as relações interativas entre os profissionais e os usuários não são as causas de maior impacto no trabalho, embora tenha revelado sobrecarga em relação a aspectos pontuais, como receio de ser agredido por um paciente e a sensação de cansaço físico ao fim do expediente. Todavia, a generalização dos resultados merece cautela, tendo em vista as especificidades de cada equipe de trabalho em cada instituição, sobretudo as diferenças regionais relacionadas às estruturas físicas e organizacionais e da rede de assistência.

Brasil. (2005). Ministério da Saúde. Secretaria de Atenção a Saúde. Coordenação Geral de Saúde Mental. Reforma psiquiátrica e política de saúde mental no Brasil. Conferência regional de reforma dos Serviços de Saúde mental: 15 anos depois de Caracas. Brasília, DF: o autor.

Camilo, C. A., Bandeira, M., Leal, R. M., \& Scalon, J. D. (2012). Avaliação da satisfação e da sobrecarga em um serviço de saúde mental. Cadernos Saúde Coletiva, 20(1), 82-92. Recuperado de http://iesc.ufrj.br/cadernos/images/csc/2012_1/artigos/CSC_v20n1_82-92.pdf

De Marco, P. F., Citero, V. A., Moraes, E., \& Nogueira-Martins, L. A. (2008). O impacto do trabalho em saúde Mental: transtornos psiquiátricos menores, qualidade de vida e satisfação profissional. Jornal Brasileiro de Psiquiatria, 57(3), 178-183. doi:10.1590/S0047-20852008000300004

Leal, R. M. A., Bandeira, M., \& Azevedo, K. R. N. (2012). Avaliação da qualidade de um serviço de saúde mental na perspectiva do trabalhador: satisfação, sobrecarga e condições de trabalho dos profissionais. Psicologia: Teoria e Prática, 14(1), 15-25. Recuperado de http://editorarevistas.mackenzie. $\mathrm{br} /$ index.php/ptp/article/view/2570

Nascimento, A. F., \& Galvanese, A. T. C. (2009). Avaliação da estrutura dos centros de atenção psicossocial do município de São Paulo, SP. Revista de Saúde Pública, 43(supl.1), 8-15. doi:10.1590/S0034-89102009000800003

Onocko-Campos, R. T., \& Furtado, J. P. (2006). Entre a saúde coletiva e a saúde mental: um instrumental metodológico para a avaliação da rede de centros de atenção psicossocial (CAPS) do Sistema Único de Saúde. Cadernos de Saúde Pública, 22(5), 1053-1062. doi:10.1590/S0102-311X2006000500018 
Pelisoli, C., Moreira, A. K., \& Kristensen, C. H. (2007). Avaliação da satisfação e do impacto da sobrecarga de trabalho em profissionais de saúde mental. Mental, 5(9), 63-78. Recuperado de http://pepsic.bvsalud.org/scielo.php?script=sci_arttext\&pi$\mathrm{d}=$ S1679-44272007000200005

Pitta, A. M. (1996). Qualidade de serviços em saúde mental: desafios para a epidemiologia. Jornal Brasileiro de Psiquiatria, 45(6), 313-321.

Rebouças, D.,Abelha, L., Legay,L. F., \&Lovisi, G.M. (2008). O trabalho em saúde mental: um estudo de satisfação e impacto. Cadernos de Saúde Pública, 24(3), 624-632. doi:10.1590/S0102-311X2008000300016

Sampaio, J. J. C., Guimarães, J. M. X., Carneiro, C., \& Garcia Filho, C. (2011). O trabalho em serviços de saúde mental no contexto da reforma psiquiátrica: um desafio técnico, político e ético. Ciência e Saúde Coletiva, 16(12), 4685-4694. doi:10.1590/S1413-81232011001300017

Santos, A. F. O., \& Cardoso, C. L. (2010). Profissionais de saúde mental: manifestação do stress e Burnout. Estudos de Psicologia, 27(1), 67-74.

Santos, A. M., Cardoso, D. A. J., \& Vieira, D. P. (2011). Análises dos níveis de satisfação de trabalhadores de saúde mental de um hospital público de referência psiquiátrica em Belém (PA). Revista Baiana de Saúde Pública, 35(4), 813-825. Reuperado de http://inseer.ibict.br/ rbsp/index.php/rbsp/article/view/256

Tenório, F. (2002). A reforma psiquiátrica brasileira, da década de 1980 aos dias atuais: história e conceitos. História, Ciências, Saúde-Manguinhos, 9(1), 25-29. doi:10.1590/S0104-59702002000100003

Vasconcellos, V. C. (2010). Trabalho em equipe na saúde mental: o desafio interdisciplinar em um CAPS. SMAD Revista Eletrônica Saúde Metal Álcool e Drogas, 6(1), 1-16. Recuperado de http:// www.revistas.usp.br/smad/article/view/38712

\section{Glaudênia Alves Moura}

Mestre pelo Programa de Pós-graduação em Saúde Coletiva da Universidade Federal do Rio Grande do Norte, Natal - RN. Brasil.

E-mail: glau_psi@yahoo.com.br

Angelo Giuseppe Roncalli

Docente da Universidade Federal do Rio Grande do Norte, Natal - RN. Brasil.

E-mail: roncalli@terra.com.br

\section{Luiz Roberto Augusto Noro}

Docente da Universidade Federal do Rio Grande do Norte, Natal - RN. Brasil.

E-mail: luiz_noro@hotmail.com

Endereço para envio de correspondência: Universidade Federal do Rio Grande do Norte. Avenida Salgado Filho, 1787 - Natal/RN, CEP: 59056-000.

Recebido: $16 / 03 / 2014$

Reformulado: 07/01/2015

Aprovado: 03/04/2016

Received:03/16/2014

Reformulated:01/07/2015

Approved:04/03/2016

Recibido: $16 / 03 / 2014$

Reformulado: 07/01/2015

Aceptado: 03/04/2016

Como citar: Moura, G. A., Roncalli, A. G. , \& Noro, L. R. A. (2016). Impacto do trabalho em profissionais de serviços de saúde mental em um município do nordeste brasileiro. Psicologia: Ciência e Profissão, 36(2): 401-410. doi:10.1590/1982-3703000342014

How to cite: Moura, G. A., Roncalli, A. G. , \& Noro, L. R. A. (2016). Work impact on professionals from mental health services in a Brazilian northeast city. Psicologia: Ciência e Profissão, 36(2): 401-410. doi:10.1590/1982-3703000342014

Cómo citar: Moura, G. A., Roncalli, A. G. , \& Noro, L. R. A. (2016). Impacto laboral sobre profesionales de servicios de salud mental en una ciudad del nordeste brasileño. Psicologia: Ciência e Profissão, 36(2): 401-410. doi:10.1590/1982-3703000342014 